\section{Pericarditis constrictiva como presentación grave e infrecuente de lupus eritematoso sistémico: reporte de un caso y revisión de la literatura}

\author{
NICOLE LUSTIG ${ }^{1, a}$, PABLO FLORENZANO ${ }^{1,2}$, \\ LUIS MANUEL SANHUEZA ${ }^{3}$, XIMENA CID $^{1, \mathrm{a}}$, GERMÁN RAMOS 3 , \\ NATALIE THONE ${ }^{\mathrm{b}}$, DEMIAN A FULLERTON ${ }^{4}$
}

\section{Constrictive pericarditis as a serious and rare presentation of systemic lupus erythematosus. Report of one case}

Constrictive Pericarditis (CP) is an unusual disease. Its most common causes are idiopathic or secondary to cardiac surgery. Less frequently it is caused by connective tissue diseases. We report a 30 years old woman hospitalized due to progressive dyspnea, chest pain and signs of right sided heart failure. During her stay, a Systemic Lupus Erythematosus (SLE) was diagnosed. The echocardiogram suggested a CP and the diagnosis was confirmed by cardiac catheterization. Pericardiectomy was successfully performed. The biopsy confirmed a nonspecific chronic pericarditis, with extensive fibrosis and absence of caseating granulomas. The patient had a satisfactory recovery.

(Rev Med Chile 2014; 142: 1065-1068)

Key words: Lupus erythematosus, systemic; Pericarditis, constrictive; Thoracic surgery.

\author{
${ }^{1}$ Escuela de Medicina Pontificia \\ Universidad Católica de Chile. \\ ${ }^{2}$ Servicio de Medicina Interna \\ Hospital Dr. Sótero del Río, \\ Santiago de Chile. \\ ${ }^{3}$ Servicio de Cardiología Hospital \\ Dr. Sótero del Río, Santiago de \\ Chile. \\ ${ }^{4}$ Servicio de Cardiocirugía \\ Hospital Dr. Sótero del Río, \\ Santiago de Chile. \\ aecada de Medicina Interna, \\ Pontificia Universidad Católica \\ de Chile. \\ bInterna de Medicina $7^{\circ}$ año, \\ Pontificia Universidad Católica \\ de Chile. \\ Recibido el 11 de septiembre de \\ 2013, aceptado el 18 de agosto \\ de 2014.
Correspondencia a: Dra. Nicole Lustig F. Departamento de Medicina Interna, Escuela de Medicina Pontificia Universidad Católica de Chile.
Lira 44, Santiago, Chile. Código postal 8330023 nmlustig@uc.cl

I a pericarditis constrictiva es una patología de baja prevalencia y difícil diagnóstico, entre $\checkmark$ sus etiologías más frecuentes se encuentra la tuberculosis y las causas idiopáticas, extrañamente se asocia a enfermedades autoinmunes complicando su evolución. Comunicamos un caso clínico de pericarditis constrictiva como complicación grave y rara de Lupus eritematoso sistémico.

\section{Caso clínico}

Mujer de 30 años con poliartritis simétrica de pequeñas y medianas articulaciones, de 6 meses de evolución, manejada como artritis reumatoídea con metotrexato y prednisona. Consultó en Urgencias del Hospital Dr. Sótero del Río, por dos semanas de disnea progresiva, palpitaciones y edema generalizado asociados a precordalgia opresiva intensa. Refirió notable disminución de masa muscular los últimos 3 meses, apareciendo también alopecia, fotosensibilidad y úlceras orales.

$\mathrm{Al}$ ingreso destacó pálida, polipneica sin desaturación, taquicárdica, normotensa y con ingurgitación yugular. Murmullo pulmonar abolido bibasal, tonos cardiacos apagados, sin soplos. Abdomen distendido, con congestión visceral, ascitis e importante edema periférico generalizado.

Al laboratorio destacaba anemia, linfopenia, hipoalbuminemia y alteración de pruebas hepáticas, sin alteración de la función renal, ni proteinuria significativa.

El electrocardiograma (ECG) mostró taquicardia sinusal y complejos de bajo voltaje. Un angio TAC de tórax descartó tromboembolismo pulmonar, revelando derrame pleural bilateral, engrosamiento pericárdico, ascitis, edema de partes 
blandas y reflujo de contraste a venas cava inferior y suprahepáticas. El análisis demostró transudado pleural y un gradiente de albúmina $>1,1$ en líquido ascítico. Por sus estigmas reumatológicos se determinaron niveles de $\mathrm{C} 3$ y C4, demostrando hipocomplementemia, anti-DNA y ANA positivos, estableciendo el diagnóstico definitivo de LES. Ante la sospecha de compromiso cardiaco asociado, se realizó ecocardiograma transtorácico, que sugirió constricción pericárdica, confirmada luego por cateterismo cardiaco.

Intentamos manejo médico con diuréticos y betabloqueadores junto a inmunosupresores con corticosteroides, hidroxicloroquina y azatioprina, por cuatro semanas, con pobre respuesta, persistiendo en anasarca, emaciación calórico-proteica progresiva e hipoalbuminemia sugerentes de enteropatía perdedora de proteínas. Se decide finalmente realizar pericardiectomía, cirugía efectuada con soporte de bypass cardiopulmonar, exitosamente. Tras la cirugía, hubo mejoría progresiva del estado general y capacidad funcional, resolución de la anasarca y los otros estigmas de falla cardiaca derecha. Las pruebas hepáticas y albuminemia se normalizaron, logrando su egreso hospitalario y retorno a domicilio en condiciones satisfactorias.

La biopsia pericárdica evidenció pericarditis crónica inespecífica, extensa fibrosis, sin granulomas caseificantes. PCR para tuberculosis fue negativa.

\section{Discusión}

El compromiso pericárdico crónico por LES ha sido comunicado anecdóticamente ${ }^{1-5}$, siendo mucho más frecuente el derrame pericárdico como manifestación de serositis aguda en el contexto de LES activo. No obstante ello, en este caso se llegó a la determinación de LES como unidad diagnóstica etiológica, a partir del estudio de un síndrome edematoso y hallazgo de constricción pericárdica, llamando la atención sobre dicha infrecuente asociación.

Para el marco teórico de fundamentación, se realizó una exhaustiva búsqueda en PubMed a través de descriptores $\mathrm{MeSH}$, considerando todos los artículos publicados hasta agosto de 2013 que incluían la asociación entre LES y PC. Adicionalmente, se buscaron artículos que incluyeran dicha asociación, contenidos en las bases de datos LILACS y SciELO.
A continuación se exponen, en primer término, algunas definiciones y consideraciones relevantes respecto a la PC y el LES como entidades independientes, para luego desarrollar la asociación clínica entre ambas condiciones.

En la PC hay pérdida de la elasticidad normal pericárdica por fibrosis y adherencias, produciendo compresión cardiaca y dificultad al llenado ${ }^{6}$, presentándose falla cardiaca predominantemente derecha, con hipertensión venosa y congestión visceral más que pulmonar ${ }^{7}$. La constricción simétrica pericárdica eleva y ecualiza las presiones diastólicas de las cuatro cámaras y la presión capilar pulmonar por aumento en la presión telediastólica ventricular, con disminución del gasto cardiaco y de la presión arterial ${ }^{8}$. El impedimento al retorno venoso explica el edema periférico.

La clínica es inespecífica, siendo los signos más relevantes: ingurgitación yugular $(96 \%)$, hepatomegalia $(92 \%)$, disnea $(77 \%)$, ascitis $(71 \%)$, distención abdominal (71\%), edema periférico $(67 \%)^{9}$. Se ha asociado también a enteropatía perdedora de proteínas por congestión venosa visceral crónica.

Para el diagnóstico destacan la ecocardiografía y el cateterismo cardiaco, sin existir un test gold-standard ${ }^{10}$. Los hallazgos ecocardiográficos frente a alta sospecha, podrían ser definitivos ocasionalmente ${ }^{11}$.

Éstos pueden resumirse en una fase de llenado diastólico precoz muy rápida, con cese abrupto e indemnidad relativa de la distensibilidad miocárdica.

Destacan al ecocardiograma bidimensional:

1. Rebote septal.

2. Interdependencia ventricular.

3. Plétora de vena cava inferior.

\section{El Doppler evidencia:}

1. Patrones característicos del llenado auricular derecho $(\mathrm{AD})$ e izquierdo (AI).

2. Variación respiratoria del llenado ventricular derecho (VD) e izquierdo (VI).

3. Variación respiratoria en el tiempo de relajación isovolumétrica.

Estos reflejan alteraciones hemodinámicas intracardiacas por limitación extrínseca al llenado, destacando la sobreexpresión del llenado diastólico precoz con cese abrupto (velocidad E de flujo transvalvular prominente, tiempo de 
desaceleración corto y velocidad A de contracción auricular muy pequeña)

Característico son las marcadas variaciones respiratorias recíprocas $(>25 \%)$ en estas velocidades de llenado entre el VD y el VI, con aumento inspiratorio de las velocidades en VD y aumento espiratorio en VI (Figura 1), faltando en la miocardiopatía restrictiva (MR), siendo útil para el diagnóstico diferencial. El Doppler tisular también demuestra mayor velocidad de llenado diastólico $\operatorname{precoz}\left(\mathrm{E}^{\prime}\right)$, a diferencia de la $\mathrm{MR}$, donde la $\mathrm{E}^{\prime}$ está marcadamente disminuida ${ }^{12}$.

El cateterismo cardiaco demuestra patrones hemodinámicos característicos en aurícula derecha: "signo de la W", que traduce elevadas presiones de contracción y llenado auricular y descenso precoz marcado al vaciarse al ventrículo. En ventrículos, se observa el característico "Dip plateau" o "signo de la raíz cuadrada", que traduce flujos diastólicos precoces rápidos con pronta ecualización y elevación de las presiones de llenado en las cuatro cámaras ${ }^{10}$ (Figura 2).

Existen múltiples etiologías de PC, siendo comunes la tuberculosis y causas idiopáticas en países subdesarrollados. En países desarrollados predominan la cardiocirugía y la radioterapia ${ }^{9,13,14}$. El manejo médico es sólo sintomático, siendo tratamiento definitivo la pericardiectomía ${ }^{15}$, cirugía no exenta de morbilidad. El trabajo de Gopalda y colaboradores reunió a 13.593 pacientes sometidos a pericardiectomía por distintas causas, de las que el 28\% correspondió a PC, describiendo mortalidad de $8 \%$ y morbilidad de $40 \%$. En PC, la principal causa de mortalidad es el bajo gasto cardiaco en el postoperatorio inicial; especialmente ante falla cardiaca derecha avanzada ${ }^{16}$. Técnicamente existen distintos abordajes (toracotomía anterolateral izquierda vs esternotomía), siendo
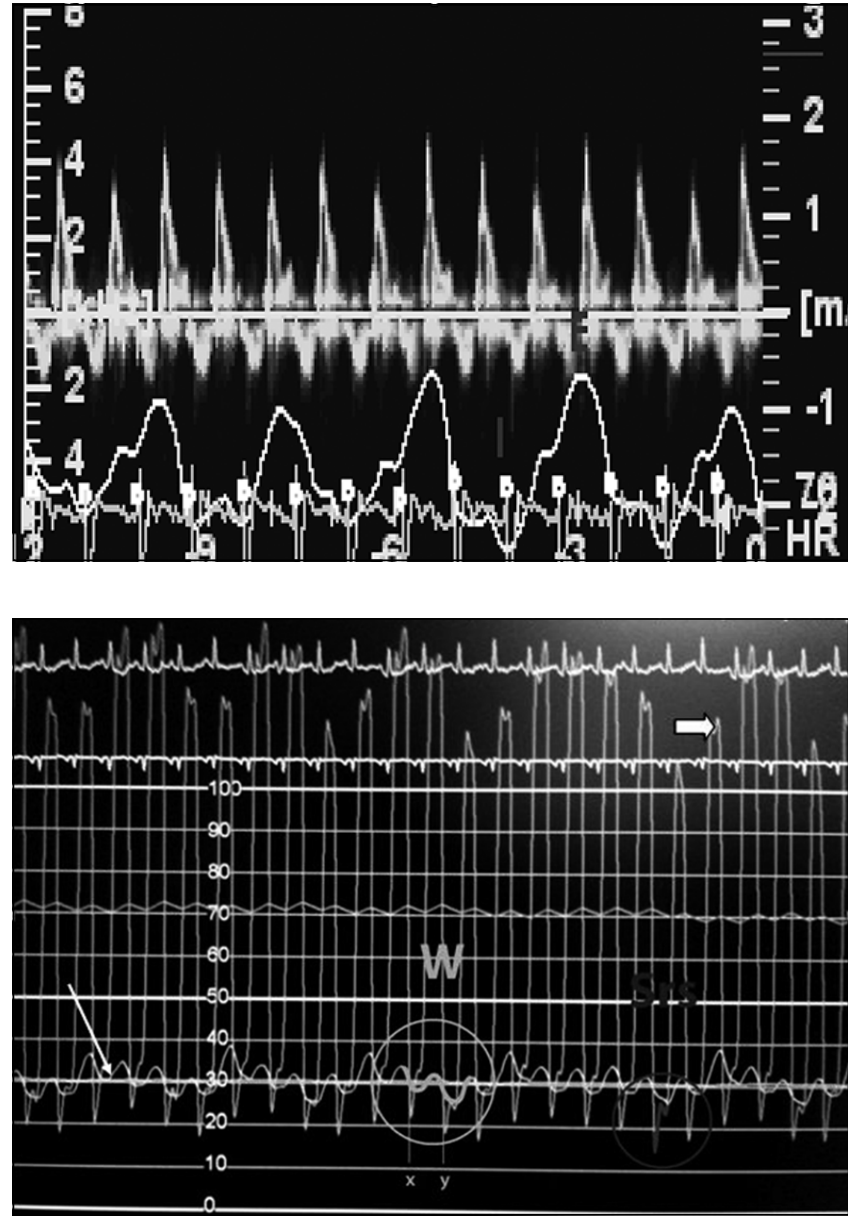

Figura 1. Eco doppler pulsado del flujo transmitral. Se observa variación respiratoria en el llenado diastólico del ventrículo izquierdo de nuestra paciente con pericarditis constrictiva. Hay disminución de la velocidad de flujo transmitral en el primer latido tras la inspiración, con reducción mayor al 25\% comparada con las velocidades máximas. E: expiración: I: inspiración .

Figura 2. Cateterismo cardiaco derecho. Curva de presión de la aurícula derecha en pericarditis constrictiva (flecha delgada). La depresión $X$ preservada y la depresión $Y$ prominente, contribuyen a la clásica onda auricular en forma de W. La curva de presión del ventrículo derecho en pericarditis constrictiva (flecha gruesa), muestra el llenado diastólico precoz rápido y el subsecuente cese abrupto del flujo debido al pericardio rígido, generando la clásica onda en dip plateau o signo de la raíz cuadrada (Srs). 
a nuestro juicio la esternotomía quien ofrece más comodidad para acceder a todo el pericardio ${ }^{17}$.

El LES es una enfermedad inflamatoria sistémica autoinmune criptogénica, caracterizada por formación de autoanticuerpos y complejos autoinmunes. Los sitios más frecuentemente afectados son la piel, articulaciones, riñones, pulmones, sistema nervioso central y serosas. El compromiso cardiovascular es bastante común, descrito en más del $50 \%$, pudiendo comprometer miocardio, endocardio, válvulas cardiacas, arterias coronarias y pericardio, siendo éste el más común (prevalencia 12-48\%, según método diagnóstico) ${ }^{1}$. Lo más frecuente es la pericarditis aguda y la efusión, siendo inhabitual el taponamiento cardiaco. En el líquido pericárdico se ha descrito presencia de ANA, células LE (phagocytic cells containing nuclei), complemento bajo e inmunocomplejos similares a los del líquido pleural en LES. La biopsia pericárdica puede revelar inflamación crónica con predominio de mononucleares, aunque lo más común es fibrosis ${ }^{18}$. La PC por LES es extremadamente infrecuente, existiendo sólo 5 casos publicados desde $1965^{1-5}$. El diagnóstico de PC se confirmó con cateterismo cardiaco y las biopsias pericárdicas mostraron fibrosis e inflamación crónica, sin granulomas. Sin embargo, es difícil establecer certeramente relación causal entre LES y PC por lo infrecuente de esta manifestación.

Dicho esto, consideramos relevante comunicar este caso de PC pesquisado como manifestación índice de LES, enfatizando que la sospecha clínica, el estudio complementario y manejo multidisciplinario, oportunos, fueron claves para evitar la progresión de una complicación potencialmente mortal.

\section{Referencias}

1. Jacobson EJ,Reza MJ. Constrictive pericarditis in systemic lupus erythematosus. Demonstration of immunoglobulins in the pericardium. Arthritis Rheum 1978; 21 (8): 972-4.

2. Starkey RH, Hahn BH. Rapid development of constrictive pericarditis in a patient with systemic lupus erythematosus. Chest 1973; 63 (3): 448-50.

3. Yurchak PM, Levine SA, Gorlin R. Constrictive Pericarditis Complicating Disseminated Lupus Erythematosus. Circulation 1965; 31: 113-8.

4. George TJ, Arnaoutakis GJ, Beaty CA, Kilic A, Baumgart- ner WA, Conte JV. Contemporary etiologies, risk factors, and outcomes after pericardiectomy. Ann Thorac Surg 2012; 94 (2): 445-51.

5. McCaughan BC, Schaff HV, Piehler JM, Danielson GK, Orszulak TA, Puga FJ, et al. Early and late results of pericardiectomy for constrictive pericarditis. J Thorac Cardiovasc Surg 1985; 89 (3): 340-50.

6. Myers RB, Spodick DH, Constrictive pericarditis: clinical and pathophysiologic characteristics. Am Heart J 1999; 138 (2 Pt 1): 219-32.

7. Mookadam F, Jiamsripong P, Raslan SF, Panse PM, Tajik AJ. Constrictive pericarditis and restrictive cardiomyopathy in the modern era. Future Cardiol 2011; 7 (4): 471-83.

8. Gaasch WH, Peterson KL, Shabetai R. Left ventricular function in chronic constrictive pericarditis. Am J Cardiol 1974; 34 (1): 107-10.

9. Ling LH, Oh JK, Schaff HV, Danielson GK, Mahoney DW, Seward JB, et al. Constrictive pericarditis in the modern era: evolving clinical spectrum and impact on outcome after pericardiectomy. Circulation 1999; 100 (13): 1380-6.

10. Kwon DH. Pericardial Disease. En Manual of Cardiovascular Medicine. Wilkins EL, Editor. 2009: Philadelphia. 393-414.

11. Talreja DR, Nishimura RA, Oh JK, Holmes DR. Constrictive pericarditis in the modern era: novel criteria for diagnosis in the cardiac catheterization laboratory. J Am Coll Cardiol 2008; 51(3): 315-9.

12. Otto C. Pericardial Disease. En Textbook of Clinical Echocardiography. E. Saunders-Elsevier, Editor. 2009: Philadelphia. 242-58.

13. Cameron J, Oesterle SN, Baldwin JC, Hancock EW. The etiologic spectrum of constrictive pericarditis. Am Heart J 1987; 113 (2 Pt 1): 354-60.

14. Bertog SC, Thambidorai SK, Parakh K, Schoenhagen P, Ozduran V, Houghtaling PL, et al. Constrictive pericarditis: etiology and cause-specific survival after pericardiectomy. J Am Coll Cardiol 2004; 43 (8): 1445-52.

15. Clare GC, Troughton RW. Management of constrictive pericarditis in the 21st century. Curr Treat Options Cardiovasc Med 2007; 9(6): 436-42.

16. Imazio M. Pericardial involvement in systemic inflammatory diseases. Heart 2011; 97 (22): 1882-92.

17. McMechan SR, McClements BM, McKeown PP, Webb SW, Adgey AA. Systemic lupus erythematosus presenting as effuso-constrictive pericarditis. Postgrad Med J 1995; 71 (840): 627-9.

18. Wolf RE, King JW, Brown TA. Antimyosin antibodies and constrictive pericarditis in lupus erythematosus. J Rheumatol 1988; 15 (8): 1284-7. 